\title{
On the Spherically Symmetric Solution to the Mixed Problem for a Weakly Hyperbolic Equation of Second Order
}

\author{
Dedicated to Professor T. Kakita on his 70th birthday
}

By

\section{Akisato $\mathrm{KUBO}^{*}$}

\section{Introduction}

We consider the following mixed problem in the domain $(0, T) \times \Omega$ :

$$
\begin{gathered}
P[v(t, x)]=f(t,|x|), \\
v=0 \quad \text { on }(0, T) \times \partial \Omega, \\
v=0, \quad v_{t}=0 \quad \text { at } t=0,
\end{gathered}
$$

where $\Omega=\left\{x \in \mathbb{R}^{n} ;|x|<1\right\}$,

$$
P[v]=v_{t t}-\sum_{i=1}^{n}\left(a(t,|x|) v_{x_{t}}\right)_{x_{t}}+\sum_{i=1}^{n} b_{i}(t, x) v_{x_{t}}+b_{0}(t,|x|) v_{t}+c(t,|x|) v,
$$

all the coefficients of $P$ are real valued and $a(t,|x|) \geq 0$ for all $(t, x) \in[0, T] \times \bar{\Omega}$. In order to obtain the spherically symmetric solution of $(0.1)-(0.3), P$ is reduced to the following operator for $r \in(0,1)=I, r=\left(x_{1}^{2}+\cdots+x_{n}^{2}\right)^{1 / 2}$ and $V(t,|x|)=$ $v(t, x)$.

$$
P_{r}[V]=V_{t t}-\left(a(t, r) V_{r}\right)_{r}+\sum_{i=1}^{n} \frac{1}{r}\left(x_{i} b_{i}(t, x)-(n-1) a(t, r)\right) V_{r}+b_{0}(t, r) V_{t}+c(t, r) V
$$

where $a(t, r), b_{0}(t, r), c(t, r)$ are in $\mathscr{B}^{\infty}([0, T] \times \bar{I})$. We impose the assumption on $P_{r}$ :

Communicated by T. Kawai, May 14, 1999.

1991 Mathematics Subject Classification(s): 35L15, 35L20, 35L80

* Partially supported by Grant-in-Aid for Scientific Research, No. 10640191, The Ministry of Education, Science, Sport and Culture. Department of Mathematics, School of Health Sciences, Fujita Health University Toyoake, Aichi 470-1192, Japan 
(A-i) $r^{-1}\left(\sum_{i=1}^{n} x_{i} b_{i}(t, x)-(n-1) a(t,|x|)\right)$ is a radial function in $x$ and belongs to $\mathscr{B}^{\infty}([0, T] \times \bar{I})$ as a function in $(t, r)$.

$(0.1)-(0.3)$ is reduced to the following problem:

$$
\begin{gathered}
P_{r}[V(t, r)]=f(t, r) \quad \text { in }(0, T) \times I, \\
V_{r}(t, 0)=V(t, 1)=0, \\
V(0, r)=0, \quad V_{t}(0, r)=0 .
\end{gathered}
$$

Therefore in order to obtain the solution of $(0.1)^{\prime}-(0.3)^{\prime}$ it is enough to solve the following mixed problem:

$$
\begin{gathered}
L_{r}[u(t, r)]=f(t, r) \quad \text { in }(0, T) \times I, \\
u_{r}(t, 0)=u(t, 1)=0, \\
u(0, r)=0, \quad u_{t}(0, r)=0,
\end{gathered}
$$

where $L_{r}[u]=u_{t t}-\left(a(t, r) u_{r}(t, r)\right)_{r}+b(t, r) u_{r}+b_{0}(t, r) u_{t}+c(t, r) u$ and $b(t, r) \in$ $\mathscr{B}^{\infty}([0, T] \times \bar{I})$. We further impose the following assumptions.

(A-ii) (Oleinik [14]) For a positive constant $t_{0} \leq T$ it holds that

$$
\alpha t b^{2}(t, r) \leq A a(t, r)+a_{t}(t, r),
$$

where $\alpha>(2 p+6)^{-1}$ ( $p$ being an integer $\left.\geq 0\right), A$ is some constant if $0 \leq t \leq t_{0}$, and $\alpha$ and $A$ are some positive constants if $t_{0} \leq t \leq T$.

(A-iii) 1) $a(t, 0)=a_{r}(t, 0)=0$.

2) There exists an extension function $\tilde{a}(t, r) \in \mathscr{B}^{2}([0, T] \times R)$ such that $\tilde{a} \geq 0$ and $\tilde{a}(t, r)=a(t, r)$ in $[0, T] \times \bar{I}$.

(A-iv) It holds that $\partial_{t}^{j} f(t, r)=0$ at $t=0, j=0,1, \ldots, p$ and $\partial_{r}^{i} f(t, r)=0$, $i=0, \ldots, s-1$ on $r=0$ for an even number $s$.

Remark 1. If $b_{i}(t, x) \equiv 0, i=0, \ldots, n, \quad(\mathrm{~A}-\mathrm{i})$ is satisfied because of (A-iii)-1). An example of the case where $a(t, r)$ and $b_{i}(t, x) \not \equiv 0, i=1, \ldots, n$ satisfy (A-i), (A-ii) and (A-iii) will be discussed in the last section in details.

Remark 2. If (A-ii) and (A-iii)-1) are satisfied, the assumption (A-iv) is seemed to be natural in the following sence. It is well known that the mixed problem for $L_{r}$ with the Dirichlet condition is reduced to the following type of problem by the usual argument, if initial data, boundary data and the forcing term are sufficiently smooth in $[0, T], \bar{I}$ and $[0, T] \times \bar{I}$ respectively and appropriate compatibility conditions are satisfied (cf. [5], [7], [14]).

$$
(M) \begin{cases}L_{r}[u]=F_{0}(t, r), & \\ u=0 & \text { on }(0, T) \times \partial I, \\ u=0, u_{t}=0 & \text { at } t=0,\end{cases}
$$


where $F_{0}(t, r)$ is sufficiently smooth in $[0, T] \times \bar{I}$ and satisfies the former part of (A-iv). Then we can find an appropriate function $U(t, r)$ so that $L_{r}[u-U]$ $=F_{0}(t, r)-L_{r}[U]=F_{1}(t, r)$ satisfies (A-iv) and $u-U$ satisfies (0.5) and (0.6). This argument will be discussed in Appendix in details.

We give simple examples of the equation (0.1).

$$
\begin{gathered}
v_{t t}-|x|^{2 k} t^{2 l} \Delta v(t, x)+d(|x|)|x|^{k-1} t^{l-1} \sum_{i=1}^{n} x_{i} v_{x_{t}}, \quad(t, x) \in(0, T) \times \Omega, \\
d(r) \in \mathscr{B}^{\infty}(\bar{I}), \quad k \in N=\{1,2, \ldots\}, \quad l \in Z_{+}=\{0,1, \ldots\},
\end{gathered}
$$

where especially we put $t^{l-1} \equiv 1$ for $l=0$ in the third term. EbiharaKawashima-Levine [2] obtained the spherically symmetric solution of the mixed problem for $v_{t t}-|x|^{2 k} \Delta v+|v|^{\alpha} v=0$ for $\alpha>0$. This type of equation is the wave operator describing a model of wave phenomenon, on or through inhomogenous medium, especially which is extremely dense near the center $(x=0)$ and then the speed of the wave vanishes near the center.

Also $a(t,|x|)$ further admits the following degeneracy on the boundary.

ii)

$$
\begin{aligned}
& a(t,|x|)=|x|^{2 k} t^{2 l}(|x|-1)^{2 \kappa}(1-|x|+t)^{2 \theta}, \quad(t, x) \in(0, T) \times \Omega \\
& \quad \text { for } k \in N, \quad \kappa, l \text { and } \theta \in \mathbb{Z}_{+} .
\end{aligned}
$$

i) is reduced to the following equation:

$$
V_{t t}-r^{2 k} t^{2 l} V_{r r}+\left(r^{k} t^{l-1} d(r)-(n-1) r^{2 k-1} t^{2 l}\right) V_{r} .
$$

Also examples of $L_{r}$ corresponding to ii) are given as follows.

ii) $)^{\prime} a(t, r)=r^{2 k} t^{2 l}(r-1)^{2 \kappa}(1-r+t)^{2 \theta}, \quad b(t, r)=r^{k} t^{l-1}(r-1)^{\kappa}(1-r+t)^{\theta}$ where especially we put $t^{l-1} \equiv 1$ for $l=0$ in $b(t, r)$.

O. A. Oleinik [14] considered the Cauchy problem to weakly hyperbolic equation of second order admitting general degeneracy in $(t, x) \in[0, T] \times \mathbb{R}^{n}$ :

$$
v_{t t}-\sum_{i, j=1}^{n}\left(A_{i j}(t, x) v_{x_{t}}\right)_{x_{j}}+\sum_{i=1}^{n} B_{i}(t, x) v_{x_{t}}+B_{0}(t, x) v_{t}+C(t, x) v=F(t, x)
$$

assuming that the following inequalities hold

$$
\begin{aligned}
\alpha t\left(\sum_{i=1}^{n} B_{i}(t, x) \xi_{i}\right)^{2} & \leq A\left(\sum_{i, j=1}^{n} A_{i j}(t, x) \xi_{i} \xi_{j}+\sum_{i, j=1}^{n} A_{i j t}(t, x) \xi_{i} \xi_{j}\right), \\
0 & \leq \sum_{l, j=1}^{n} A_{i j}(t, x) \xi_{i} \xi_{j}
\end{aligned}
$$


for any $\xi \in \mathbb{R}^{n}$ where all the coefficients of $(0.8)$ are sufficiently smooth and bounded. Note that $(0.7)$ is the special case of $(0.9)$. Then she proved that there exists the smooth solution. Her result was extended to the equation of higher order by Menikoff [10] and Ohya [11].

The well-posedness of the mixed problem for regularly hyperbolic equations of second order was proved by Ikawa [3], [4]. But nothing is known about the mixed problem corresponding to Oleinik's result except for the simple degeneracy case. In fact, Kimura [5] restricted degeneracy of (0.8) to the case where $A_{i j}$ and $B_{i}$ degenerate in $t$ only of polynomial order (cf. Chi Min-you [1]) and impose the null Dirichlet boundary condition in a bounded domain in $\mathbb{R}^{n}$ with compact smooth boundary. Then she proved the well-posedness of the problem (cf. [7]). The mixed problems of a weakly hyperbolic equations of second order with other kind of degeneracy was studied by [8] and [9]. On the other hand, Krasnov [6] and Oleinik [12] showed the existence theorems and uniqueness theorems in the sense of a generalized solution to the mixed problem for weakly hyperbolic equations under some conditions on the coefficients and data.

Our purpose is to obtain the smooth spherically symmetric solution of the mixed problem $(0.1)-(0.3)$ corresponding to the Cauchy problem considered in Oleinik [14]. Let us introduce some notations:

$$
\begin{gathered}
(h, g)_{I(\tau)}=\int_{0}^{\tau} \int_{I} h(t, r) g(t, r) d r d t, \quad(h, g)_{I}(t)=\int_{I} h(t, r) g(t, r) d r \\
\|h\|_{I, k}(t)=\left\{\sum_{i+j \leq k}\left(\partial_{r}^{j} \partial_{t}^{i} h, \partial_{r}^{j} \partial_{t}^{i} h\right)_{I}(t)\right\}^{1 / 2}, \quad\|h\|_{I(\tau), k}=\left\{\int_{0}^{\tau}\|h\|_{I, k}^{2}(t) d t\right\}^{1 / 2}, \\
\|h\|_{I ; q, s, k}(t)=\left\{\sum_{i \leq q, j \leq s, q+s \leq k}\left(\partial_{r}^{j} \partial_{t}^{i} h, \partial_{r}^{j} \partial_{t}^{i} h\right)_{I}\right\}^{1 / 2}, \\
\|h\|_{I(\tau) ; q, s, k}=\left\{\sum_{i \leq q, j \leq s, q+s \leq k}\left(\partial_{r}^{j} \partial_{t}^{i} h, \partial_{r}^{j} \partial_{t}^{i} h\right)_{I(\tau)}\right\}^{1 / 2}, \\
\partial_{t}=\frac{\partial}{\partial t}, \quad \partial_{r}=\frac{\partial}{\partial r}, \quad \partial_{x_{t}}=\frac{\partial}{\partial x_{i}}, \quad i=1, \ldots, n, \quad D_{x}^{\alpha}=\partial_{x_{1}}^{\alpha_{1}} \ldots \partial_{x_{n}}^{\alpha_{n}},
\end{gathered}
$$

where $\alpha=\left(\alpha_{1}, \ldots, \alpha_{n}\right)$ is a multi-index. Let $\lambda_{j}, j=1,2, \ldots$, be eigen values of $-\partial_{r}^{2}$ with the null Dirichlet condition such that $0<\lambda_{1}<\lambda_{2}<\cdots$ and corresponding eigen functions $\varphi_{1}(r), \varphi_{2}(r), \ldots$ We define a functional space

$$
V^{(k)}(I)=\left\{v(r)=\sum_{i=1}^{\infty} g_{i} \varphi_{i}(r) ; \sum_{i=1}^{\infty} \lambda_{i}^{2 k} g_{i}^{2}<\infty, r \in \mathbb{I}\right\} .
$$


It is well known that $V^{(k)}(I) \subset \stackrel{\circ}{H}^{1}(I) \cap H^{k}(I)$ and that the following inequality holds. For some positive constants $c_{i}, i=1,2$ and $v(r) \in V^{(k)}(I)$ it holds that

$$
c_{1}\|v\|_{I, k}^{2} \leq \sum_{i=1}^{\infty} g_{i}^{2} \lambda_{i}^{2 k} \leq c_{2}\|v\|_{I, k}^{2} .
$$

Theorem 1. Let $f(t, r)$ be in $\bigcap_{i=0}^{s+p+1} C^{i}\left([0, T] ; V^{(s+p+1-i)}(I)\right)$ for any even number s. Assume that (A-ii)-(A-iv) hold. Then there eixists a unique solution $u(t, r) \in \bigcap_{i=0}^{s} C^{i}\left([0, T] ; V^{(s-i)}(I)\right)$ of the problem $(0.4)-(0.6)$ and satisfies the following estimate for some constant $M>0$ and $\tau \in[0, T]$ :

$$
\|u\|_{I, s}^{2}(\tau) \leq M\left(\|f\|_{I(T) ; 0, s, s}^{2}+\|f\|_{I, s-2}^{2}(\tau)+\max _{0 \leq \sigma \leq t_{0}}\|f\|_{I ; p+1+s, s, p+1+s}^{2}(\sigma)\right)
$$

Replacing $b(t, r)$ by $\sum_{i=1}^{n} r^{-1}\left(x_{i} b_{i}(t, x)-(n-1) a(t, r)\right)$ in $(0.7)$ and going back to the original problem through $(0.1)^{\prime}-(0.3)^{\prime}$, we have the following result.

Theorem 2. Let $f(t, r) \in \bigcap_{i=0}^{s+1+p} C^{i}\left([0, T] ; V^{(s+p+1-i)}(I)\right)$ for any even number s. Assume that (A-i), (A-iii), (A-iv) and (A-ii) replaced by $\sum_{i=1}^{n} r^{-1}\left(x_{i} b_{i}(t, x)-(n-1) a(t, r)\right)$ instead of $b(t, r)$ in $(0.7)$ hold. Then there exists a solution $v(t, x) \in C^{s-1}([0, T] \times \bar{\Omega})$ of $(0.1)-(0.3)$.

Remark 3. (A-iii)-1) is required to prove (0.5) in Theorem 1 and to show the regularity of the solution in Theorem 2. However even if (A-iii)-1) is not satisfied, the proof of Theorem 1 guarantees the existence of a smooth solution satisfying the mixed problem $(0.4),(0.6)$ and $u(t, 0)=u(t, 1)=0$.

Now let us explain our argument. In Oleinik [14] the energy estimate played an essential role. If we try to derive the energy estimate for $(0.8),(0.2)$ and $(0.3)$ in $(0, T) \times \Omega$ following to her method in the usual Sobolev space, integrating by parts we have many remainder terms of inner products defined on the boundary. They are in very complicated forms and so harmful to deriving the energy inequality of higher order. Hence crutial point in our problem lies in treatment with them. In the first step, to simplify the form of them we restrict our attention to seeking the spherically symmetric solution of (0.4)(0.6). For this purpose $P$ is reduced to $P_{r}$ by $r=\left(x_{1}^{2}+\cdots+x_{n}^{2}\right)^{1 / 2}$. Since $r^{-1}\left(\sum_{i=1}^{n} x_{i} b_{i}(t, x)-(n-1) a(t,|x|)\right)$ may be regarded as a radial function under the assumption (A-i), it is enough to consider $L_{r}$ instead of $P_{r}$.

In the second step, we introduce the functional space $V^{(2 k)}(I)$ spanned by eigen functions of $-\partial_{r}^{2}$ satisfying null Dirichlet boundary condition. It holds that $\partial_{r}^{2 l} \varphi_{j}(r)=0$ at $r=0,1, l=0,1, \ldots, j \in \mathbb{N}$. By the use of this property most of such simplified remainder terms become harmless and we obtain the desired estimate. Consequently our main purpose in this paper will be carried 
out through $(0.1)^{\prime}-(0.3)^{\prime}$ by seeking the spherically symmetric solution of $(0.4)-$ $(0.6)$ by Galerkin's method. The energy inequality of $(0.4)-(0.6)$ plays an important role and it is derived according to Oleinik [14].

This paper is organized as follows. In the first section, we derive the basic energy inequality for (0.4)-(0.6). In section 2 , in the first subsection we obtain the energy inequality of higher order with respect to $r$. Most crutial point in this paper lies here. Next, using it, we have the energy inequality of higher order with respect to $(t, r)$. By this estimate we obtain the existence of smooth solution of (0.4)-(0.6). Finally, from this result the existence of solution of the original problem $(0.1)-(0.3)$ follows. We also give an example of $a(t,|x|)$ and $b_{i}(t, x) \not \equiv 0, i=1, \ldots, n$, satisfying (A-i)-(A-iii).

\section{§. Basic Energy Inequality \\ 1.1. Construction of Solution}

$f(t, r)$ is written in the form of $\sum_{i=0}^{\infty} f_{i}(t) \varphi_{i}(r)$ where $f_{i}(t)=\left(f(t, r), \varphi_{i}(r)\right)_{I}$. Put $f_{J}(t, r)=\sum_{i=0}^{J} f_{i}(t) \varphi_{i}(r)$ and set $u_{J}(t, r)=\sum_{i=0}^{J} g_{J i}(t) \varphi_{i}(r)$ for $J=1,2, \ldots$ Then we construct $u_{J}(t, r)$ so that $u_{\infty}(t, r)$ satisfies the problem $(0.4)-(0.6)$. For this purpose, we consider $\left(L_{r}\left[u_{J}\right], \varphi_{j}\right)_{I}=\left(f_{J}, \varphi_{j}\right)_{I}$. The left hand side of this equality is written in the form:

$$
\left(L_{r}\left[u_{J}\right], \varphi_{j}\right)_{I}=\partial_{t}^{2} g_{J j}(t)+P_{J j}\left(t ; g_{J 1}, \ldots, g_{J J}, \partial_{t} g_{J 1}, \ldots, \partial_{t} g_{J J}\right)
$$

where $P_{J j}$ is a linear operator in $g_{J 1}, \ldots, g_{J J}, \partial_{t} g_{J 1}, \ldots$ and $\partial_{t} g_{J J}$. We determine $g_{J j}(t), j=1, \ldots, J$ so that $g_{J j}(t)$ satisfies the following problem:

$$
\left\{\begin{array}{l}
\partial_{t}^{2} g_{J j}(t)+P_{J j}\left(t ; g_{J 1}, \ldots, g_{J J}, \partial_{t} g_{J 1}, \ldots, \partial_{t} g_{J J}\right)=f_{j} \\
g_{J j}=0, \quad g_{J j t}=0 \quad \text { at } t=0, j=1, \ldots, J
\end{array}\right.
$$

This problem is reduced to the following ordinary differential system in $g_{J j}$ and $G_{J j}=\partial_{t} g_{J j}, j=1, \ldots, J$ :

$$
\left\{\begin{array}{l}
\partial_{t} g_{J j}(t)=G_{J j}(t), \\
\partial_{t} G_{J j}(t)+P_{j}\left(t ; g_{J 1}, \ldots, g_{J J}, G_{J 1}, \ldots, G_{J J}\right)=f_{j}, \\
g_{J j}(0)=0, \quad G_{J j}(0)=0, \quad j=1, \ldots, J
\end{array}\right.
$$

It is well known that there is a smooth solution in $[0, T]$ of this problem. In fact coefficients of $P_{J j}$ are sufficiently smooth in $t$. Since $f_{j}(t) \in C^{p+1+s}([0, T])$, we have $g_{J j}(t) \in C^{p+3+s}([0, T])$. 


\subsection{Basic Energy Inequality for $0 \leq t \leq t_{0}$}

For simplicity, put $u_{J}(t, r)=u(t, r)$ and put $f_{J}(t, r)=f(t, r)$ again through this section and next section. Then we have

$$
\left\{\begin{array}{l}
\left(L_{r}[u], u\right)_{I}=(f, u)_{I}, \\
u(t, 0)=u(t, 1)=0 \\
u(0, r)=u_{t}(0, r)=0
\end{array}\right.
$$

In this subsection our desired estimate will be derived by the manner due to Oleinik [14]. We have the following basic energy estimate of $u$.

Lemma 1. It holds that for $0 \leq \tau \leq t_{0} \leq T$ we have for a constant $M_{1}$

$$
\|u\|_{I, 0}^{2}(\tau) \leq M_{1} \max _{0 \leq \sigma \leq t_{0}}\|f\|_{I ; p+1,0, p+1}^{2}(\sigma)
$$

supposed that (A-ii) and (A-iv) hold.

Proof. Denote $w(t, x)=\int_{t}^{\tau} u(\sigma, x) d \sigma$ for $0 \leq t \leq \tau$ according to Oleinik [14]. It is easily seen that it holds for $\theta>0$

$$
\left(L[u], e^{\theta t} w\right)_{I(\tau)}=\left(f, e^{\theta t} w\right)_{I(\tau)} .
$$

First we have

$$
\begin{aligned}
\left(u_{t t}, w e^{\theta t}\right)_{I(\tau)} & =-\left(u_{t}, w \theta e^{\theta t}\right)_{I(\tau)}+\left(u_{t}, u e^{\theta t}\right)_{I(\tau)} \\
& =\left(u,-u \theta e^{\theta t}+w \theta^{2} e^{\theta t}\right)_{I(\tau)}+\left(u_{t}, u e^{\theta t}\right)_{I(\tau)} .
\end{aligned}
$$

On the other hand it holds

$$
\left(u_{t}, u e^{\theta t}\right)_{I(\tau)}=\frac{-\left(u, u \theta e^{\theta t}\right)_{I(\tau)}+\left(u, u e^{\theta t}\right)_{I}(\tau)}{2} .
$$

Therefore we have

$$
\left(u_{t t}, w e^{\theta t}\right)_{I(\tau)}=-\frac{3}{2}\left(u, u \theta e^{\theta t}\right)_{I(\tau)}+\left(u, w \theta^{2} e^{\theta t}\right)_{I(\tau)}+\frac{1}{2}\left(u, u e^{\theta t}\right)_{I}(\tau) .
$$

Next we estimate the elliptic part.

$$
\begin{aligned}
\left(\left(a(t, r) u_{r}\right)_{r}, w e^{\theta t}\right)_{I(\tau)} & =-\left(a u_{r}, w_{r} e^{\theta t}\right)_{I(\tau)} \\
& =\left[\left(a w_{r}, w_{r} e^{\theta t}\right)_{I}\right]_{0}^{1}-\left(a w_{r}, \partial_{t}\left(w_{r} e^{\theta t}\right)\right)_{I(\tau)}-\left(a_{t} w_{r}, w_{r} e^{\theta t}\right)_{I(\tau)} .
\end{aligned}
$$

Therefore we have

$$
\left(\left(a(t, r) u_{r}\right)_{r}, w e^{\theta t}\right)_{I(\tau)}=-\frac{\left(\left(\theta a+a_{t}\right) w_{r}, w_{r} e^{\theta t}\right)_{I(\tau)}}{2}-\frac{\left(a w_{r}, w_{r}\right)_{I}(0)}{2} .
$$


Lower order terms of $L_{r}[u]$ are estimated as follows.

$$
\begin{gathered}
\left(b_{0}(t, r) u_{t}, w e^{\theta t}\right)_{I(\tau)}=\left(u, e^{\theta t} b_{0}(t, r) u-\left(b_{0}(t, r) e^{\theta t}\right)_{t} w\right)_{I(\tau)} . \\
\left(b(t, r) u_{r}, w e^{\theta t}\right)_{I(\tau)}=-\left(b_{r}(t, r) u, w e^{\theta t}\right)_{I(\tau)}-\left(b(t, r) w_{r}, u e^{\theta t}\right)_{I(\tau)} \\
\leq M_{2} \tau^{2}\left(u, t^{-1} u e^{\theta t}\right)_{I(\tau)}+\frac{1}{2}\left(\alpha t b^{2}(t, r) w_{r}, w_{r} e^{\theta t}\right)_{I(\tau)} \\
+(2 \alpha)^{-1}\left(u, t^{-1} u e^{\theta t}\right)_{I(\tau)} .
\end{gathered}
$$

Finally we estimate the right hand side of (1.1). By integrating by parts we have

$$
\left(f, w e^{\theta t}\right)_{I(\tau)}=(-1)^{p+1}\left(\partial_{t}^{p+1} f, w_{p+1}\right)_{I(\tau)},
$$

where $w_{p+1}(t, x)=\int_{t}^{\tau} w_{p}(\sigma, x) d \sigma$ and $w_{0}=w e^{\theta t}$. Then we have

$$
\begin{aligned}
\left|w_{p+1}\right|^{2} & \leq\left|\int_{t_{p+1}}^{\tau} \ldots \int_{t_{1}}^{\tau} \int_{t_{0}}^{\tau} w e^{\theta t} d t d t_{0} \ldots d t_{p}\right|^{2} \\
& \leq\left|\tau^{p+1} e^{\theta \tau} \int_{0}^{\tau} u(t, x) d t\right|^{2} \leq \tau^{2 p+2} e^{2 \theta \tau}\left(\int_{0}^{\tau} u(t, x) d t\right)^{2} \\
& \leq \tau^{2 p+3} e^{2 \theta \tau} \int_{0}^{\tau} u^{2}(t, x) d t .
\end{aligned}
$$

Hence we have

$$
\int_{t}^{\tau}\left|w_{p+1}\right|^{2} d t \leq \tau^{2 p+4} e^{2 \theta \tau} \int_{0}^{\tau} u^{2} d t \leq \tau^{2 p+5} e^{2 \theta \tau} \int_{0}^{\tau} t^{-1} u^{2} d t
$$

Therefore we obtain for a constant $\delta>0$

$$
\left|\left(f, w e^{\theta t}\right)_{I(\tau)}\right| \leq \delta\left(u, t^{-1} u e^{\theta t}\right)_{I(\tau)}+\frac{e^{\theta t_{0}} \tau^{2 p+6}}{4 \delta} \max _{0 \leq \sigma \leq t_{0}}\|f\|_{I ; p+1,0, p+1}^{2}(\sigma) .
$$

Put $\theta=A$, for $\tau \leq t_{0}$ and set $y(\tau)=\left(u, t^{-1} u e^{\theta t}\right)_{I(\tau)}$. Then taking account of (A-ii) we have

$$
\tau y^{\prime}(\tau) \leq\left(\alpha^{-1}+2 \delta\right) y(\tau)+M_{3} \tau y(\tau)+M_{4} \tau^{2 p+6} \max _{0 \leq \sigma \leq t_{0}}\|f\|_{I ; p+1,0, p+1}^{2}(\sigma) .
$$

Term by term multiplying by $\tau^{-\alpha^{-1}-2 \delta} e^{-M_{3} \tau}$

$$
\begin{aligned}
y(\tau) & \leq \tau^{\alpha^{-1}+2 \delta} e^{M_{3} \tau} \int_{0}^{\tau}\left(M_{4} e^{-M_{3} \sigma} \max _{0 \leq \theta \leq t_{0}}\|f\|_{I ; p+1,0, p+1}^{2}(\theta) \sigma^{2 p+6-\alpha^{-1}-2 \delta}\right) d \sigma \\
& \leq M_{4} \tau^{2 p+6} \max _{0 \leq \sigma \leq t_{0}}\|f\|_{I ; p+1,0, p+1}^{2}(\sigma) .
\end{aligned}
$$

This completes Lemma 1. 國 


\section{§2. Energy Inequality of Higher Order}

In this section we derive the energy inequality of higher order for $L_{r}[u]$, provided that (A-ii), (A-iii)-2) and (A-iv) hold.

\subsection{Energy Inequality of Higher Order in $r$-Derivatives for $0 \leq t \leq t_{0}$}

In this subsection, we derive the energy estimate to $r$-derivatives of $u$ of higher order. For this purpose we prepare the following two lemmas.

Lemma 2.1. For $k=0,1,2, \ldots$, we have

$$
\left(\partial_{r}^{2 k+1} u, \partial_{r}^{2 k+1} u\right)_{I, 0} \leq \frac{1}{2}\left(\left\|\partial_{r}^{2 k} u\right\|_{I, 0}^{2}+\left\|\partial_{r}^{2 k+2} u\right\|_{I, 0}^{2}\right) .
$$

Proof. Since $\partial_{r}^{2 k} u=0$ at $r=0,1$, we have

$$
\left(\partial_{r}^{2 k+1} u, \partial_{r}^{2 k+1} u\right)_{I, 0}=\left[\partial_{r}^{2 k} u \partial_{r}^{2 k+1} u\right]_{0}^{1}-\left(\partial_{r}^{2 k} u, \partial_{r}^{2 k+2} u\right)_{I, 0}=-\left(\partial_{r}^{2 k} u, \partial_{r}^{2 k+2} u\right)_{I, 0} .
$$

By Cauchy-Schwarz's inequality we have the desired result.

Lemma 2.2. Suppose that $g(r) \geq 0, r \in \boldsymbol{R}$ and $g(r) \in \mathscr{B}^{2}(\boldsymbol{R})$. Then we have

$$
\left(\partial_{r} g(r)\right)^{2} \leq 2\left\{\sup _{r \in R}\left|g_{r r}\right|\right\} g(r), \quad r \in \boldsymbol{R} .
$$

Proof. The proof is done according to Oleinik [13, Lemma 4]. Suppose that (2.1) is not satisfied at a point $r_{0} \in \boldsymbol{R}$, i.e., $g_{r}^{2}\left(r_{0}\right)>2\left\{\sup _{r \in R}\left|g_{r r}\right|\right\} g\left(r_{0}\right)$. Consider a point $\tilde{r}_{0}=r_{0}-2 \frac{g\left(r_{0}\right)}{g_{r}\left(r_{0}\right)}$. By Taylor's formula for a point $\bar{r}$ between $\tilde{r}_{0}$ and $r_{0}$ we have

$$
g\left(\tilde{r}_{0}\right)=g\left(r_{0}\right)-2 \frac{g\left(r_{0}\right)}{g_{r}\left(r_{0}\right)} g_{r}\left(r_{0}\right)+2 \frac{g^{2}\left(r_{0}\right)}{g_{r}^{2}\left(r_{0}\right)} g_{r r}(\bar{r})=-g\left(r_{0}\right)\left(1-2 \frac{g\left(r_{0}\right)}{g_{r}^{2}\left(r_{0}\right)} g_{r r}(\bar{r})\right) .
$$

In view of the hypotheses $1-2 \frac{g\left(r_{0}\right)}{g_{r}^{2}\left(r_{0}\right)} g_{r r}(\bar{r})>0$ holds. If $g\left(r_{0}\right)>0$, then it holds $g\left(\tilde{r}_{0}\right)<0$, which contradicts $g(r) \geq 0$. In case $g\left(r_{0}\right)=0, g_{r}^{2}\left(r_{0}\right)>0$ holds, which contradicts $g(r) \geq 0$ in a neighborhood of $r_{0}$. Hence we proved that (2.1) holds for $r \in \boldsymbol{R}$.

Lemma 2.1 implies that to carry out our aim in this subsection it is enough to derive the inequality for derivatives of even order of $u$ with respect to $r$. Put $y_{v}(\tau)=\left(\partial_{r}^{v} u, e^{\theta_{1} t} t^{-1} \partial_{r}^{v} u\right)_{I(\tau)}$. We have the following result.

Lemma 2.3. There exists a constant $M_{5}$ and a positive constant $\theta_{1}$ such that we have for $v \leq s$ and $\tau \in\left[0, t_{0}\right]$

$$
y_{v}(\tau) \leq M_{5} \tau^{2 p+6} \max _{0 \leq \sigma \leq t_{0}}\|f\|_{I ; p+1, s, p+1+s}^{2}(\sigma) .
$$


Proof. Recall that $s$ is any even number. Suppose that the following inequality holds for $v \leq s-2$ for a constant $E_{v}$.

$$
y_{v}(\tau) \leq E_{v} \tau^{2 p+6} \sum_{q \leq \nu} \max _{0 \leq \sigma \leq t_{0}}\left\|\partial_{r}^{q} f\right\|_{I ; p+1,0, p+1}^{2}(\sigma) .
$$

In the same way as derived (1.2) we have for a positive constant $\theta_{1}$

$$
\left(\partial_{r}^{s} u_{t t}, \partial_{r}^{s} w e^{\theta_{1} t}\right)_{I(\tau)}=\frac{1}{2}\left(\partial_{r}^{s} u, \partial_{r}^{s} u e^{\theta_{1} t}\right)_{I}(\tau)+\left(\partial_{r}^{s} u e^{\theta_{1} t}, \theta_{1}^{2} \partial_{r}^{s} w-\frac{3 \theta_{1}}{2} \partial_{r}^{s} u\right)_{I(\tau)} .
$$

Estimating the elliptic part we have

$$
\begin{aligned}
\left(\partial_{r}^{s}\left(a(t, r) u_{r}\right)_{r}, \partial_{r}^{s} w e^{\theta_{1} t}\right)_{I(\tau)}= & \left(\left(a(t, r) \partial_{r}^{s} u_{r}\right)_{r}, \partial_{r}^{s} w e^{\theta_{1} t}\right)_{I(\tau)} \\
& +\sum_{v \leq s} C_{v}\left(\partial_{r}^{v} a \partial_{r}^{s-v} u_{r}, e^{\theta_{1} t} \partial_{r}^{s} w_{r}\right)_{I(\tau)}=I+I I
\end{aligned}
$$

where $C_{v}$ is a constant. Then we have in the same way as derived (1.3)

$$
I=-\frac{1}{2}\left(\left(\theta_{1} a+a_{t}\right) \partial_{r}^{s} w_{r}, \partial_{r}^{s} w_{r} e^{\theta_{1} t}\right)_{I(\tau)}-\frac{1}{2}\left(a \partial_{r}^{s} w_{r}, \partial_{r}^{s} w_{r}\right)_{I}(0)
$$

In the case of $v=1$ in $I I$, we have

$$
\begin{aligned}
& \left(\left(\partial_{r} a\right) \partial_{r}^{s-1} u_{r}, e^{\theta_{1} t} \partial_{r}^{s} w_{r}\right)_{I(\tau)} \\
& =\left(\left(\partial_{r} a\right) \partial_{r}^{s-1} w_{r}, e^{\theta_{1} t} \partial_{r}^{s} w_{r}\right)_{I}(0)+\left(\left(\partial_{t} \partial_{r} a\right) \partial_{r}^{s-1} w_{r}, e^{\theta_{1} t} \partial_{r}^{s} w_{r}\right)_{I(\tau)} \\
& \quad+\left(\left(\partial_{r} a\right) \partial_{r}^{s-1} w_{r}, \theta_{1} e^{\theta_{1} t} \partial_{r}^{s} w_{r}\right)_{I(\tau)}-\left(\left(\partial_{r} a\right) \partial_{r}^{s-1} w_{r}, e^{\theta_{1} t} \partial_{r}^{s} u_{r}\right)_{I(\tau)}
\end{aligned}
$$

considering that $s$ is an even number

$$
\begin{aligned}
= & -\frac{1}{2}\left(\left(\partial_{r}^{2} a\right) \partial_{r}^{s-1} w_{r}, e^{\theta_{1} t} \partial_{r}^{s} w\right)_{I}(0)-\frac{1}{2}\left(\left(\partial_{t} \partial_{r}^{2} a\right) \partial_{r}^{s-1} w_{r}, e^{\theta_{1} t} \partial_{r}^{s} w\right)_{I(\tau)} \\
& -\frac{1}{2}\left(\left(\partial_{r}^{2} a\right) \partial_{r}^{s-1} w_{r}, \theta_{1} e^{\theta_{1} t} \partial_{r}^{s} w\right)_{I(\tau)}+\left(\left(\partial_{r} a\right) \partial_{r}^{s-1} w_{r r}, e^{\theta_{1} t} \partial_{r}^{s} u\right)_{I(\tau)} \\
& +\left(\left(\partial_{r}^{2} a\right) \partial_{r}^{s-1} w_{r}, e^{\theta_{1} t} \partial_{r}^{s} u\right)_{I(\tau)} \\
\leq & -\frac{\left(\left(\partial_{r}^{2} a\right) \partial_{r}^{s-1} w_{r}, e^{\theta_{1} t} \partial_{r}^{s} w\right)_{I}(0)}{2}+\left(\left(\partial_{r} a\right) \partial_{r}^{s-1} w_{r r}, e^{\theta_{1} t} \partial_{r}^{s} u\right)_{I(\tau)}+\left|A_{1}\right|
\end{aligned}
$$

Here as well as below we denote by $A_{j}$ the integrals which can be estimated in the following way: $\left|A_{j}\right| \leq N_{j} \sum_{v \leq s} \tau\left(\partial_{r}^{v} u, t^{-1} \partial_{r}^{v} u e^{\theta_{1} t}\right)_{I(\tau)}$ for some constants $N_{j}>0$. By Lemma 2.2 and (A-iii)-2) we have

$$
\begin{aligned}
& \left(\left(\partial_{r} a\right) \partial_{r}^{s-1} w_{r r}, e^{\theta_{1} t} \partial_{r}^{s} u\right)_{I(\tau)} \\
& \quad \leq\left(a\left(\partial_{r}^{s-1} w\right)_{r r}, e^{\theta_{1} t}\left(\partial_{r}^{s-1} w\right)_{r r}\right)_{I(\tau)}+\text { const. }\left(e^{\theta_{1} t} \partial_{r}^{s} u, \partial_{r}^{s} u\right)_{I(\tau)} .
\end{aligned}
$$


On the other hand

$$
\begin{aligned}
- & \frac{\left(\left(\partial_{r}^{2} a\right) \partial_{r}^{s-1} w_{r}, e^{\theta_{1} t} \partial_{r}^{s} w\right)_{I}(0)}{2} \\
= & \int_{0}^{\tau} \partial_{t} \frac{\left(\left(\partial_{r}^{2} a\right) \partial_{r}^{s-1} w_{r}, e^{\theta_{1} t} \partial_{r}^{s} w\right)_{I}(t)}{2} d t \\
= & \frac{\left(\left(\partial_{r}^{2} \partial_{t} a\right) \partial_{r}^{s-1} w_{r}, e^{\theta_{1} t} \partial_{r}^{s} w\right)_{I(\tau)}}{2}-\frac{\left(\left(\partial_{r}^{2} a\right) \partial_{r}^{s-1} u_{r}, e^{\theta_{1} t} \partial_{r}^{s} w\right)_{I(\tau)}}{2} \\
& -\frac{\left(\left(\partial_{r}^{2} a\right) \partial_{r}^{s-1} w_{r}, e^{\theta_{1} t} \partial_{r}^{s} u\right)_{I(\tau)}}{2}+\frac{\left(\left(\partial_{r}^{2} a\right) \partial_{r}^{s-1} w_{r}, \theta_{1} e^{\theta_{1} t} \partial_{r}^{s} w\right)_{I(\tau)}}{2} .
\end{aligned}
$$

Hence we have

$$
\left(a_{r} \partial_{r}^{s-1} u_{r}, e^{\theta_{1} t} \partial_{r}^{s} w_{r}\right)_{I(\tau)} \leq\left(a\left(\partial_{r}^{s-1} w\right)_{r r}, e^{\theta_{1} t}\left(\partial_{r}^{s-1} w\right)_{r r}\right)_{I(\tau)}+\left|A_{2}\right| .
$$

Now let us estimate the case of $v \geq 2$ in $I I$

$$
\begin{aligned}
& \sum_{s \geq v \geq 2} C_{v}\left(\left(\partial_{r}^{v} a\right) \partial_{r}^{s-v} u_{r}, e^{\theta_{1} t} \partial_{r}^{s} w_{r}\right)_{I(\tau)} \\
& =-\sum_{s \geq v \geq 2} C_{v}\left(\left(\partial_{r}^{v} a\right) \partial_{r}^{s-v} u_{r r}, e^{\theta_{1} t} \partial_{r}^{s} w\right)_{I(\tau)} \leq\left|A_{3}\right|
\end{aligned}
$$

Combining above these inequalities we have

$$
I I \leq\left(a\left(\partial_{r}^{s-1} w\right)_{r r}, e^{\theta_{1} t}\left(\partial_{r}^{s-1} w\right)_{r r}\right)_{I(\tau)}+\left|A_{4}\right| .
$$

On the other hand we have

$$
\left(\partial_{r}^{s}\left(b(t, r) u_{r}\right), e^{\theta_{1} t} \partial_{r}^{s} w\right)_{I(\tau)} \leq\left|\left(b(t, r) \partial_{r}^{s} u, e^{\theta_{1} t} \partial_{r}^{s} w_{r}\right)_{I(\tau)}+A_{5}\right|
$$

in the same way as derived (1.5)

$$
\leq \frac{\alpha}{2} t\left(b^{2}(t, r) \partial_{r}^{s} w_{r}, e^{\theta_{1} t} \partial_{r}^{s} w_{r}\right)_{I(\tau)}+(2 \alpha)^{-1}\left(\partial_{r}^{s} u, t^{-1} e^{\theta_{1} t} \partial_{r}^{s} u\right)_{I(\tau)}+\left|A_{5}\right| .
$$

Other lower order terms are estimated as follows.

$$
\begin{aligned}
& \left(\partial_{r}^{s}\left(b_{0}(t, r) u_{t}\right), \partial_{r}^{s} w e^{\theta_{1} t}\right)_{I(\tau)} \\
& \quad=\left(\partial_{r}^{s}\left(b_{0} u\right), e^{\theta_{1} t} \partial_{r}^{s}\left(u-\theta_{1} w\right)\right)_{I(\tau)}-\left(\partial_{r}^{s}\left(b_{0 t} u\right), e^{\theta_{1} t} \partial_{r}^{s} w\right)_{I(\tau)}=A_{6} .
\end{aligned}
$$

Finally, choosing $\theta_{1}$ sufficiently large we have by using Lemma 2.1 and (2.3) for a positive constant $C$

$$
\tau y_{s}^{\prime}(\tau) \leq\left(\alpha^{-1}+2 \delta\right) y_{s}(\tau)+C \tau y_{s}(\tau)+M_{6} \tau^{2 p+6} \sum_{\nu \leq s} \max _{0 \leq \sigma \leq t_{0}}\left\|\partial_{r}^{v} f\right\|_{I ; p+1,0, p+1}^{2}(\sigma) .
$$

Thus we have in the same way as derived (1.6) 


$$
y_{s}(\tau) \leq M_{7} \tau^{2 p+6} \sum_{v \leq s} \max _{0 \leq \sigma \leq t_{0}}\left\|\partial_{r}^{v} f\right\|_{I ; p+1,0, p+1}^{2}(\sigma)
$$

Considering into Lemma 2.1 we see that (2.2) holds.

\subsection{Energy Inequality of Higher Order for $0 \leq t \leq \mathbb{t}_{0}$}

In this subsection, we derive the energy estimate of higher order derivatives of $u(t, r)$ in $t$ and $r$.

Lemma 2.4. There exists a constant $M_{8}>0$ such that it holds for $\tau \in\left[0, t_{0}\right]$

$$
\|u\|_{I, s}^{2}(\tau) \leq M_{8}\left(\|f\|_{I\left(t_{0}\right) ; 0, s-1, s-1}^{2}+\|f\|_{I, s-2}^{2}(\tau)+\max _{0 \leq \sigma \leq t_{0}}\|f\|_{I ; p+1, s, p+s+1}^{2}(\sigma)\right) .
$$

Proof. In the case of $v \leq s-1$, we have for a positive constant $\theta_{2}$

$$
\left(\partial_{r}^{v} u_{t t}, e^{-\theta_{2} t} \partial_{r}^{v} u_{t}\right)_{I(\tau)}=\frac{1}{2}\left(\partial_{r}^{v} u_{t}, e^{-\theta_{2} t} \partial_{r}^{v} u_{t}\right)_{I}(\tau)+\frac{1}{2}\left(\partial_{r}^{v} u_{t}, \theta_{2} e^{-\theta_{2} t} \partial_{r}^{v} u_{t}\right)_{I(\tau)}
$$

Next we have

$$
\begin{aligned}
\left(\partial_{r}^{v}\left(a(t, r) u_{r}\right)_{r}, \partial_{r}^{v} u_{t} e^{-\theta_{2} t}\right)_{I(\tau)} & \\
= & \frac{1}{2}\left(\left(-\theta_{2} a+a_{t}\right) \partial_{r}^{v} u_{r}, \partial_{r}^{v} u_{r} e^{-\theta_{2} t}\right)_{I(\tau)}-\frac{1}{2}\left(a \partial_{r}^{v} u_{r}, \partial_{r}^{v} u_{r}\right)(\tau) \\
& +\sum_{1 \leq k \leq v} C_{k}\left(\left(\partial_{r}^{k} a \partial_{r}^{v-k} u_{r}\right)_{r}, e^{-\theta_{2} t} \partial_{r}^{v} u_{t}\right)_{I(\tau)}
\end{aligned}
$$

On the other hand we have

$$
\begin{gathered}
\left(\partial_{r}^{v}\left(b(t, r) u_{r}\right), e^{-\theta_{2} t} \partial_{r}^{v} u_{t}\right)_{I(\tau)} \leq\left|\left(\partial_{r}^{v} u_{t}, e^{-\theta_{2} t} \partial_{r}^{v} u_{t}\right)_{I(\tau)}\right|+M_{9} \sum_{v+1 \geq k}\left(\partial_{r}^{k} u, e^{-\theta_{2} t} \partial_{r}^{k} u\right)_{I(\tau)}, \\
\left(\partial_{r}^{v}\left(b_{0}(t, r) u_{t}\right), e^{-\theta_{2} t} \partial_{r}^{v} u_{t}\right)_{I(\tau)} \leq M_{10} \sum_{v \geq k}\left(\partial_{r}^{k} u_{t}, e^{-\theta_{2} t} \partial_{r}^{k} u_{t}\right)_{I(\tau)}
\end{gathered}
$$

Taking Lemma 2.3 into account and choosing $\theta_{2}$ sufficiently large we have

$$
\begin{aligned}
& \sum_{v \leq s-1}\left(\partial_{r}^{v} u_{t}, \partial_{r}^{v} u_{t}\right)_{I}(\tau) \\
& \quad \leq M_{11}\left(\tau^{2 p+6} \max _{0 \leq \sigma \leq t_{0}} \sum_{v \leq s}\left\|\partial_{r}^{v} f\right\|_{I ; p+1,0, p+1}^{2}(\sigma)+\sum_{\nu \leq s-1}\left(\partial_{r}^{v} f, \partial_{r}^{v} f\right)_{I\left(t_{0}\right)}\right) .
\end{aligned}
$$

In order to estimate the derivatives of the form: $\partial_{r}^{v} \partial_{t}^{\rho+2} u, \rho \geq 0, v+\rho \leq s-2$, we apply the operator $\partial_{r}^{v} \partial_{t}^{\rho}$ to the equation (0.4) and we obtain

$$
\partial_{r}^{v} \partial_{t}^{\rho+2} u=\partial_{r}^{v} \partial_{t}^{\rho}\left(L_{r}[u]-\partial_{t}^{2} u\right) .
$$


Since in the right hand side of (2.7) derivatives of $u$ are in the form: $\partial_{r}^{i} \partial_{t}^{j} u$, $i+j \leq s, j \leq \rho+1$, by using (2.7) we arrive at (2.5).

\subsection{Energy Inequality of Higher Order for $0 \leq t \leq \mathbb{T}$}

We may obtain the desired estimate for $\tau \in\left[t_{0}, T\right]$ in a similar way as in the above. But the estimate for $\left(b(t, r) u_{r}, w e^{\theta t}\right)_{I(\tau)}$ should be derived as in the following.

$$
\begin{aligned}
\left(b(t, r) u_{r}, w e^{\theta t}\right)_{I(\tau)}= & -\left(b_{r}(t, r) u, w e^{\theta t}\right)_{I(\tau)}-\left(b(t, r) u, w_{r} e^{\theta t}\right)_{I(\tau)} \\
\leq & M_{12}\left(u, e^{\theta t} u\right)_{I(\tau)}+\frac{\alpha}{2}\left(t b^{2}(t, r) w_{r}, w_{r} e^{\theta t}\right)_{I\left(t_{0}\right)} \\
& +(2 \alpha)^{-1}\left(u, t^{-1} e^{\theta t} u\right)_{I\left(t_{0}\right)}+\frac{\alpha_{1}}{2}\left(b^{2}(t, r) w_{r}, w_{r} e^{\theta t}\right)_{I\left(t_{0}, \tau\right)} \\
& +\left(2 \alpha_{1}\right)^{-1}\left(u, u e^{\theta t}\right)_{I\left(t_{0}, \tau\right)}
\end{aligned}
$$

where $\alpha_{1}$ is a constant and $\alpha_{1} \leq \alpha t$ for $t \geq t_{0}$ and $I\left(t_{0}, \tau\right)=\left(t_{0}, \tau\right) \times I$.

Set $z=\left(u, u e^{\theta t}\right)_{I(\tau)}$ for $t_{0} \leq \tau \leq T$. By using the estimate (1.6) for $y\left(t_{0}\right)=$ $\left(u, t^{-1} u e^{\theta t}\right)_{I\left(t_{0}\right)}$ we have for sufficiently large $\theta$ and $\tau>t_{0}$

$$
\begin{aligned}
z^{\prime}(\tau) & \leq M_{13}\left(z(\tau)+y\left(t_{0}\right)+(f, f)_{I(T)}\right) \\
& \leq M_{13}\left(z(\tau)+(f, f)_{I(T)}\right)+M_{14} \max _{0 \leq \sigma \leq t_{0}}\|f\|_{I ; p+1,0, p+1}^{2}(\sigma) .
\end{aligned}
$$

Therefore we have

$$
(u, u)_{I(\tau)} \leq M_{15}\left(\max _{0 \leq \sigma \leq t_{0}}\|f\|_{I ; p+1,0, p+1}^{2}(\sigma)+(f, f)_{I(T)}\right) .
$$

Using the estimate (2.2), in the same way we estimate $\left(\partial_{r}^{v} u, \partial_{r}^{v} u\right)(\tau)$ for $v \leq$ $s$ in case $\tau>t_{0}$. The derivatives of the form $\partial_{r}^{v} \partial_{t}, v \leq s-1$ and $\partial_{r}^{v} \partial_{t}^{\rho+2}, \rho \geq 0$, $v+\rho \leq s-2$ for $\tau>t_{0}$ are estimated in the same way as for $\tau \leq t_{0}$. Then we have the following result.

Lemma 2.5. We have for some constant $M_{16}>0$ and $0 \leq \tau \leq T$

$$
\|u\|_{I, s}^{2}(\tau) \leq M_{16}\left(\|f\|_{I(T) ; 0, s, s}^{2}+\|f\|_{I, s-2}^{2}(\tau)+\max _{0 \leq \sigma \leq t_{0}}\|f\|_{I ; p+1, s, p+1+s}^{2}(\sigma)\right) .
$$

\section{§3. The Proof of Theorems}

\subsection{The Proof of Theorems}

First we discuss the convergence of formal series $\left\{f_{J}\right\}_{J=1}^{\infty}$.

Lemma 3.1. It holds that 
i) $f_{J}(t, r) \rightarrow f(t, r)$ strongly in $\bigcap_{i=0}^{p+1+s} C^{i}\left([0, T] ; V^{p+1+s-i}(I)\right)$ as $J \rightarrow \infty$,

ii) $f_{J}(t, r) \rightarrow f(t, r)$ strongly in $H^{s-1}((0, T) \times I)$ as $J \rightarrow \infty$.

Proof. i) Taking (0.10) into account, it holds that for $t \in[0, T]$

$$
\begin{aligned}
\left\|f_{J}\right\|_{I, p+1+s}^{2}(t) & =\sum_{i+j \leq p+1+s} \sum_{l=1}^{J}\left(\partial_{t}^{i} f_{l}\right)^{2} \lambda_{l}^{2 j} \\
& \rightarrow \sum_{i+j \leq p+1+s} \sum_{l=1}^{\infty}\left(\partial_{t}^{i} f_{l}\right)^{2} \lambda_{l}^{2 j}=\|f\|_{I, p+1+s}^{2}(t) \quad \text { as } J \rightarrow \infty .
\end{aligned}
$$

Since $\max _{0 \leq t \leq T}\|f\|_{I, p+1+s}^{2}(t)<+\infty$, we have

$$
\max _{0 \leq t \leq T}\left\|f-f_{J}\right\|_{I, p+1+s}^{2}(t)=\max _{0 \leq t \leq T} \sum_{i+j \leq p+1+s} \sum_{l=J}^{\infty}\left(\partial_{t}^{i} f_{l}\right)^{2} \lambda_{l}^{2 j} \rightarrow 0 \quad \text { as } J \rightarrow \infty .
$$
theorem

ii) Considering into the proof of i) we have by Lebesgue's convergence

$$
\int_{0}^{T}\left\|f-f_{J}\right\|_{I, s-1}^{2}(\sigma) d \sigma \rightarrow 0
$$

Hence we proved ii).

Proof of Theorem 1. For any $J_{1}, J_{2} \in N$ with $J_{1} \geq J_{2}$, from Lemma 2.5 it follows that $u_{J_{1}}-u_{J_{2}}$ satisfies

$$
\begin{aligned}
\left\|u_{J_{1}}-u_{J_{2}}\right\|_{I, s}(\tau) \leq & M_{17}\left(\left\|f_{J_{1}}-f_{J_{2}}\right\|_{I(T) ; 0, s, s}^{2}+\left\|f_{J_{1}}-f_{J_{2}}\right\|_{I, s-1}^{2}(\tau)\right. \\
& \left.+\max _{0 \leq \sigma \leq t_{0}}\left\|f_{J_{1}}-f_{J_{2}}\right\|_{I ; p+1, s, p+1+s}^{2}(\sigma)\right) .
\end{aligned}
$$

Lemma 3.1 yields that the right hand side of (3.1) tends to zero as $J_{1}, J_{2} \rightarrow \infty$. Hence there exists a limiting function $u(t, r)$ such that

$$
u_{J} \rightarrow u \text { strongly in } \bigcap_{i=0}^{s} C^{i}\left([0, T] ; V^{s-i}(I)\right) \quad \text { as } J \rightarrow \infty .
$$

Since $f_{J} \rightarrow f$ in $L^{2}((0, T) \times I)$, for any $w(t, x) \in \mathscr{D}((0, T) \times I)$ we have

$$
\begin{aligned}
\left(L_{r}\left[u_{J}\right], w\right)_{(0, T) \times I} & =\left(f_{J}, w\right)_{(0, T) \times I} \\
\rightarrow\left(L_{r}[u], w\right)_{(0, T) \times I} & =(f, w)_{(0, T) \times I} \quad \text { as } J \rightarrow \infty .
\end{aligned}
$$

It is easily seen that

$$
u(t, r)=0 \quad \text { on } r=0,1 \text {. }
$$


From (A-ii) and (A-iii)-1) $b(t, 0)=0$ follows for $t \in[0, T]$. Put $v_{j}(t)=\partial_{r}^{j} u(t, 0)$, $j=0,1,2, \ldots$ Suppose that $v_{j}=0, j=0,1, \ldots, k-1$ for $s-1 \geq k$. Since $\left(\partial_{r}^{i} f\right)(t, 0)=0, i=0,1, \ldots, s-1$, differentiating the both sides of $(0.4)$ in $r$ of order $k$, we have for $t \in[0, T]$, taking (A-iii)-1) into account,

$$
\left\{\begin{array}{c}
\partial_{t}^{2} v_{k}+\sum_{i=0}^{k} C_{i}\left\{\left(\partial_{r}^{i} b\right)(t, 0) v_{k+1-i}+\left(\partial_{r}^{i} b_{0}\right)(t, 0) \partial_{t} v_{k-i}+\left(\partial_{r}^{i} c\right)(t, 0) v_{k-i}\right\} \\
=\partial_{t}^{2} v_{k}+k b_{r}(t, 0) v_{k}+b_{0}(t, 0) \partial_{t} v_{k}+c(t, 0) v_{k}=0 \\
v_{k}=v_{k t}=0 \quad \text { at } t=0
\end{array}\right.
$$

Then we have $v_{k}(t) \equiv 0$. Therefore we obtain

$$
\partial_{r}^{i} u=0, \quad i=0,1, \ldots, s-1 \quad \text { on } r=0 .
$$

Also the estimate (3.1) implies that (0.11) holds for $u(t, r)$. Thus the function $u(t, r)$ is the desired solution of $(0.4)-(0.6)$.

The proof of Theorem 2. By Theorem 1 we obtain the solution $V(t, r)$ of $(0.1)^{\prime}-(0.3)^{\prime}$ in $\bigcap_{i=0}^{s} C^{i}\left([0, T] ; V^{s-i}(I)\right) \cap C^{s-1}([0, T] \times \bar{I})$. Repeating the same procedure from (3.2) to (3.3) we have

$$
\partial_{r}^{i} \partial_{t}^{j} V(t, r)=0, \quad i+j \leq s-1 \quad \text { on } r=0 .
$$

Also (3.4) implies that for the solution $V(t, r)$ of $(0.1)^{\prime}-(0.3)^{\prime}$ the derivatives of $V(t,|x|)$ in $x$ up to the order $s-1$ are continuous at $x=0$. In fact, we have

$$
\sum_{|\alpha| \leq s-1}\left|D_{x}^{\alpha} V(t,|x|)\right| \leq C \sum_{i+j \leq s-2} r^{-j}\left|\left(\partial_{r}^{i+1} V\right)(t,|x|)\right|
$$

by Ohya [11, Lemma 14.1$]$

$$
<C \max _{0 \leq r \leq 1}\left|\left(\partial_{r}^{s-1} V\right)(t, r)\right|<+\infty .
$$

Similarly we have $\max _{x \in \bar{\Omega}} \sum_{|\alpha|+j \leq s-1}\left|\partial_{t}^{j} D_{x}^{\alpha} V(t,|x|)\right|<+\infty$. Therefore $V(t,|x|)$ is our desired solution (0.1)-(0.3).

\subsection{Example of $a(t,|x|)$ and $b_{i}(t, x)$}

Recall that in Theorem 2 (A-ii) is assumed to hold for $\sum_{i=1}^{n}$ $r^{-1}\left(x_{i} b_{i}(t, x)-(n-1) a(t, r)\right)$ instead of $b(t, r)$ in $(0.7)$. In this subsection we give an example of $b_{i}(t, x)$ and $a(t, r)$ satisfying (A-i), (A-iii)-1) and (0.7) replaced by $\sum_{i=1}^{n} r^{-1}\left(x_{i} b_{i}(t, x)-(n-1) a(t, r)\right)$ instead of $b(t, r)$.

Assume that $a(t, r)=r^{2} \eta(t, r)$ for a function $\eta(t, r)(\geq 0) \in \mathscr{B}^{\infty}([0, T] \times \bar{I})$, which satisfies (A-iii)-1). Let $\beta(t, r)$ belong to $\mathscr{B}^{\infty}([0, T] \times \bar{I})$. Define $b_{i}(t, x)=x_{i} \beta / r, \quad i=1, \ldots, n$. Then we have $\sum_{i=1}^{n} b_{i}(t, x) \partial_{x_{l}}=\beta(t, r) \partial_{r}$. Therefore $(\mathrm{A}-\mathrm{i})$ is satisfied and $P_{r}$ is written in the form: 


$$
P_{r}\left[V_{]}=V_{t t}-\left(a(t, r) V_{r}\right)_{r}+(\beta(t, r)-r(n-1) \eta(t, r)) V_{r}+b_{0}(t, r) V_{t}+c(t, r) V .\right.
$$

For a constant $A^{\prime}>0$ we see that $a(t, r)$ satisfies $\left(r^{-1}(n-1) a\right)^{2} \leq A^{\prime} a$. We assume that $a(t, r)$ and $\beta(t, r)$ satisfy

$$
2 \alpha t \beta^{2} \leq\left(A-2 A^{\prime}\right) a+a_{t}
$$

where $\alpha>(2 p+6)^{-1}$ ( $p$ being an integer $\left.\geq 0\right), A-2 A^{\prime}$ is some constant if $0 \leq t \leq t_{0}$, and $\alpha$ and $A-2 A^{\prime}$ are some positive constant if $t>t_{0}$. It is easily seen that $a(t,|x|)$ and $b_{i}(t, x)$ defined in above are our desired ones.

\section{Appendix}

Considering into (A-ii) and (A-iii)-1) we may assume that $a(t, r)$ and $b(t, r)$ are denoted by $r^{2} A(t, r)$ and $r B(t, r)$ respectively for $A(t, r)$ and $B(t, r) \in$ $\mathscr{B}^{\infty}([0, T] \times \bar{I})$. Then we have the following result:

Proposition. Assume that $F_{0}(t, r) \in \mathscr{B}^{\infty}([0, T] \times \bar{I})$ and that $\left(\partial_{t}^{i} F_{0}\right)(0, r)=$ $0, i=0, \ldots, l$ for a positive integer $l \geq 2$. For any positive integer $k \leq l / 2$, there exists a smooth function $U(t, r)$ such that for $F_{1}(t, r)=F_{0}(t, r)-L_{r}[U]$

$$
\begin{gathered}
\left(\partial_{r}^{i} F_{1}\right)(t, 0)=0, \quad i=0, \ldots, k, \\
\left(\partial_{t}^{i} F_{1}\right)(0, r)=0, \quad i=0, \ldots, l-2 k .
\end{gathered}
$$

Proof. Note that

$$
L_{r}=\partial_{t}^{2}-r^{2} A(t, r) \partial_{r}^{2}-r^{2} A_{r}(t, r) \partial_{r}+r(B(t, r)-2 A(t, r)) \partial_{r}+b_{0}(t, r) \partial_{t}+c(t, r) .
$$

Define operators $L_{r}^{1, i}$ and $L_{r}^{2, i}, i=0, \ldots, k$ as in the following.

$$
\left\{\begin{array}{l}
L_{r}^{1,0}=\partial_{t}^{2}+b_{0}(t, r) \partial_{t}+c(t, r) \\
L_{r}^{1,1}=\partial_{t}^{2}+b_{0}(t, r) \partial_{t}+c(t, r)+(B(t, r)-2 A(t, r)), \\
L_{r}^{1, i}=\partial_{t}^{2}+b_{0}(t, r) \partial_{t}+c(t, r)-i(i-1) A(t, r)+i(B(t, r)-2 A(t, r)), \quad i \geq 2, \\
\quad r^{i+1} L_{r}^{2, i}[\cdot]=L_{r}\left[r^{i} \cdot\right]-r^{i} L_{r}^{1, i}[\cdot], \quad i \geq 0 .
\end{array}\right.
$$

Then we consider the Cauchy problem of the following ordinary differential equation for $i=0, \ldots, k$ :

$$
(L i)\left\{\begin{array}{l}
L_{r}^{1, i}\left[w_{i}(t, r)\right]=\left(\partial_{r}^{i} F_{0}\right)(t, 0)-i L_{r}^{2, i-1}\left[w_{i-1}\right], \\
w_{i}=w_{i t}=0 \quad \text { at } t=0,
\end{array}\right.
$$

where $L_{r}^{2,-1} \equiv 0$. We see that there exists a unique smooth solution $w_{i}(t, r)$ of $(L i)$ (see Kubo [8, Proposition 1.1]). It is seen that $\left(\partial_{t}^{j} w_{i}\right)(0, r)=0$, $j=0, \ldots, l+2-2 i$. Put $U(t, r)=\sum_{j=0}^{k} w_{j}(t, r) \rho(r) r^{j} / j$ ! for a non-negative 
function $\rho(r) \in \mathscr{B}^{\infty}(\bar{I})$ satisfying $\rho(r)=1$ near $r=0$ and $\rho(r)=0$ near $r=1$. Then we have near $r=0$

$$
\begin{aligned}
F_{0}(t, r)-L_{r}[U] & =F_{0}(t, r)-\sum_{j=0}^{k}\left(\left(\partial_{r}^{j} F\right)(t, 0) \frac{r^{J}}{j !}-r^{j} L_{r}^{2, j-1}\left[\frac{w_{j-1}}{(j-1) !}\right]+r^{j+1} L_{r}^{2, j}\left[\frac{w_{j}}{j !}\right]\right) \\
& =F_{0}(t, r)-\sum_{j=0}^{k}\left(\partial_{r}^{j} F\right)(t, 0) \frac{r^{j}}{j !}-r^{k+1} L_{r}^{2, k}\left[\frac{w_{k}}{k !}\right] .
\end{aligned}
$$

Therefore we have $F_{1}(t, r)=O\left(r^{k+1}\right)$ and $\left(\partial_{t}^{i} F_{1}\right)(0, r)=0, j=0, \ldots, l-2 k$ hold. Hence the proof is complete.

Let $u$ be a smooth solution of $(M)$ in Remark 2. Proposition implies that $L_{r}[u-U]=F_{1}$ holds. Since it holds that $\left.L_{r}[u]\right|_{r=0}=\left.L_{r}^{1,0}[u]\right|_{r=0}$, considering into $(L 0)$ on $r=0$, from the uniqueness of the solution of $(L 0)$ restricted on $r=0 \quad w_{0}(t, 0)=u(t, 0)$ follows. Since it holds $L_{r}[u-U]=F_{1}$, repeating the procedure from (3.2) to (3.3) for $u-U$, we have $\partial_{r}^{j}(u-U)=0, j=0, \ldots, k$ on $r=0$. Thus we see that $u-U$ satisfies $(0.5)$ and $(0.6)$.

\section{Acknowledgement}

The author would like to express his sincere gratitude to Professor K. Mochizuki for his valuable criticism. Also his thanks are devoted to Professor T. Mandai for his helpful advice.

\section{References}

[1] Chi Min-you, On the Cauchy problem for a class of hyperbolic equations with initial data on the parabolic degenerating line, Acta Math. Sinica, 8 (1958), 521-529.

[2] Ebihara, Y., Kawashima, S. and Levine, H. A., On solutions to $u_{t t}-|x|^{\alpha} \Delta u=f(u)$ $(\alpha>0)$, Funk. Ekv., 38 (1995), 539-544.

[3] Ikawa, M., Mixed problem for hyperbolic equations of second order, J. Math. Soc. Japan, 20 (1968), 580-608.

[4] - On the mixed problem for hyperbolic equations of second order with the Neumann boundary condition, Osaka J. Math., 7 (1970), 203-223.

[5] Kimura, K., A mixed problem for weakly hyperbolic equations of second order, Comm. Partial Diff. Equations, 6 (1981), 1335-1361.

[6] Krasnov, M. L., The mixed boundary value problem for degenerating linear differential equations of second order, Mat. Sb., 49 (91) (1959), 29-84.

[7] Kubo, A., On the mixed problems for weakly hyperbolic equations of second order, Comm. Partial Diff. Equations, 9 (1984), 889-917.

[8] - Mixed problems for some weakly hyperbolic second order equations, Math. Japonica, 29 (1984), 721-751.

[9] Nakaoka, A., On boundary value problems for elliptic equations degenerating on the boundary, Publ. RIMS, Kyoto Univ., 7 (1971/72), 455-482. 
[10] Menikoff, A., The Cauchy problem for weakly hyperbolic equations, Amer. J. Math., 97 (1975), 548-558.

[11] Ohya, Y., Le problème de Cauchy à caractéritiques multiples, Ann. Scuola Norm. Sup. Pisa, 4 (4) (1977), 757-805.

[12] Oleinik, O. A., The Cauchy problem and the boundary value problem for second order hyperbolic equations degenerating in a domain and its boundary, Sviet Math. Dokl., 7 (4) (1966), 969-973.

[13] -, On linear equations of second order with non-negative characteristic form, Mat. Sb., N.S., 69 (111) (1966), 111-140. (in Russian.) English transl., Amer. Math. Soc. Transl.

[14] - On the Cauchy problem for weakly hyperbolic equations, Comm. Pure Appl. Math., 23 (1970), 569-586. 